\title{
Editorial: Topical Volume on Earth's Magnetic Field-Understanding Geomagnetic Sources from the Earth's Interior and Its Environment
}

\author{
Claudia Stolle $^{1}$ • Nils Olsen ${ }^{2,3}$ • Arthur D. Richmond ${ }^{4}$. \\ Hermann J. Opgenoorth
}

Received: 20 December 2016 / Accepted: 8 March 2017 / Published online: 20 March 2017

(C) Springer Science+Business Media Dordrecht 2017

The Earth's magnetic field results from different sources in the Earth's interior and in near Earth space. Commonly, when investigating solar-terrestrial interactions where the Earth's internal field plays a major role, scientists concentrate on strong (tens to thousands of nanotesla) and rapid (seconds to days) magnetic field variations that are caused by currents in the ionosphere and magnetosphere when solar activity, and correspondingly the electric currents in Earth's environment, are enhanced. However, for studying the internal sources of the geomagnetic field, originating in the core and crust, scientists use observations from so called "geomagnetic quiet" times, when external field variations are expected to be weak. However, even these weak variations impact internal field modelling, and incomplete knowledge of them hinders their separation. Difficulties arise in particular in characterizing the long term behaviour of external sources, e.g., seasonal and solar cycle variations of the magnetospheric ring current, polar convection currents or ionospheric dynamo currents driven by atmospheric tides, since they have amplitudes and spatial scales similar to those of the core field's secular variation or the lithospheric field. Since such external currents are also present during geomagnetic quiet conditions they may result in biased core and crustal field models. An additional complexity arises from magnetic observations taken by satellites because of the movement of the platform, leading to a possible space-time ambiguity.

The challenge of source separation has been recognised for several decades but no definite solution has been found so far. Questions related to proper source characterisation of the magnetic field are for example:

- How can "magnetic quiet conditions" be defined in polar and non-polar regions? Which sources are present during magnetic quiet conditions and what is their character?

\footnotetext{
C. Stolle

cstolle@gfz-potsdam.de

1 GeoForschungsZentrum, Potsdam, Germany

2 DTU Space, Technical University Denmark, Kongens Lyngby, Copenhagen, Denmark

3 National Center for Atmospheric Research, High Altitude Observatory, Boulder, CO, USA

4 Swedish Institute for Space Physics, Uppsala, Sweden
} 
- What modelling capabilities exist for predicting magnetic field contributions during magnetically non-active conditions?

- What is the uncertainty in specifying internal field contributions for studying external currents?

In total 20 articles are collected in this special issue of Space Science Reviews that are responding to these questions by addressing one of the following scientific areas: Geomagnetic classifications and observations, External field relevance for investigating the Earth's interior, Impact of the geomagnetic large-scale spatial and long-term variations for investigating near-Earth space, and State of the art and challenges in near-Earth electrodynamics during quiet times. These areas have been approached by observations: time series, that are analysed for specific magnetic signatures, models: empirical modelling results that are based on data, such as inversions, often used to describe climatological behaviours, and simulations: physics-based modelling results from first principles.

Olsen and Stolle provide an overview of electric currents in the ionosphere and magnetosphere during geomagnetic quiet times and illustrate their magnetic signature with an example of the Swarm satellite observations, focusing on its magnitude and effect on eastwest and north-south field differences. Laundal and Richmond survey the various magnetic coordinate systems used for analysis of magnetospheric and ionospheric electrodynamics, including non-orthogonal coordinate systems based on realistic models of the main geomagnetic field. Kauristie et al. discuss the distribution of and the relation between different well-established geomagnetic indices to investigate their mutual consistency in describing quiet conditions, while $M c$ Pherron and Chu propose a new mid-latitude substorm index that is derived after removing the main field and classical storm pattern and can further be used to identify quiet times. Chulliat et al. include new developments, science opportunities, and perspectives of magnetic observations on ground and in space. Finlay et al. and Thébault et al. discuss challenges in a proper handling of external sources when modelling the core and crustal field, respectively. Laundal et al. review the hemispheric differences in the magnetic field at polar latitudes for its consequences in the climatological pattern of electroand neutral dynamics, and Cnossen provides an overview on how secular variation of the geomagnetic field affects the strengths and the shape of ionospheric current systems. FriisChristensen at al. concentrate on ionospheric currents at dark quiet-time polar latitudes from ground- and space-based observations. Yamazaki and Maute review the midlatitude $S_{q}$ currents and the equatorial electrojet emphasizing on their solar-cycle, seasonal, longitudinal, and day-to-day variations. Fejer et al. review effects of the ionospheric disturbance dynamo that may last several days after a geomagnetic storm, i.e., a time which is often classified as "geomagnetic quiet" based on geomagnetic indices. $L u$ discusses the capabilities of multiinstrumental assimilative efforts to reproduce large-scale fields and currents at polar and auroral latitudes. Alken et al. review low-latitude $F$-region gravity and pressure-gradient current models. Maute and Richmond examine capabilities of simulating $F$-region currents at low and mid latitudes, while Yokoyama and Stolle discuss a new model to account for electrodynamics of nighttime plasma structures. Lühr et al. investigate the effects of magnetospheric tail and ring currents in ground and satellite magnetometer data. Milan et al. use satellite observations in the ionosphere to illustrate the variability of the field-aligned currents connecting the ionosphere with the magnetosphere in response to variations in solar radiation. The conditions under which the currents are expected to be weak are also outlined. Magnetospheric simulation models have been developed since many years and sophisticated candidates are now in operation. Wiltberger et al. discuss the present state of a magnetospheric model to reproduce as realistically as possible climatological patterns of the polar field-aligned currents. Based on predictions of another simulation model Raeder et al. 
show global geomagnetic perturbations at altitudes of low Earth orbiting satellites that arise from magnetospheric, field-aligned, and high latitude E region ionospheric currents.

By compiling this collection, we hope to provide a comprehensive view on the characterisation of the quiet-time geomagnetic environment, on advances achieved and remaining limitations and challenges, and future opportunities. 